\title{
Study of regioselective dialkylation of naphthalene in the presence of reusable zeolite catalysts
}

\author{
Keith Smith,* Simon D. Roberts and Gamal A. El-Hiti $\uparrow$ \\ Centre for Clean Chemistry, Department of Chemistry, University of Wales Swansea, \\ Singleton Park, Swansea, UK SA2 8PP
}

Received 3rd January 2003, Accepted 6th March 2003

First published as an Advance Article on the web 27th March 2003

Highly regioselective dialkylation of naphthalene using various alkylating agents can be achieved over zeolite catalysts. For example, the tert-butylation of naphthalene (1) using tert-butanol in cyclohexane over a dealuminated H-Mordenite (HM) zeolite has been optimised to give a $60 \%$ yield of 2,6-di-tert-butylnaphthalene (3) with a 2,6/2,7 ratio of over 50. This has been achieved by varying the reaction time, temperature, solvent, pressure, amount of tert-butanol, solvent and catalyst, Si/Al ratio of the catalyst, and the mode of addition. The zeolites can be easily regenerated by heating and reused.

\section{Introduction}

Recently, considerable emphasis has been placed on improvement in the environmental impact of industrial chemical processes. ${ }^{1}$ It is well recognised that solids such as zeolites can play an important role in the development of greener technologies through their abilities to act as heterogeneous catalysts, support reagents, entrain by-products, avoid aqueous work-ups and influence product selectivities. ${ }^{2}$ Advances are particularly needed in the area of electrophilic substitutions, ${ }^{3}$ where traditional Lewis acid catalysts are a cause of considerable concern and where reactions are frequently unselective. In our previous studies, we have shown that zeolites can be used successfully to overcome a number of these problems and we have used them or other solids in the development of cleaner organic reactions, including nitration, ${ }^{4}$ bromination, ${ }^{5}$ chlorination, ${ }^{6}$ acylation, allylation, ${ }^{8}$ alkylation ${ }^{9}$ and methanesulfonylation ${ }^{10}$ of aromatic compounds.

Our recent studies in this area have focussed on the regioselective dialkylation of naphthalene. ${ }^{9}$ This topic is of great interest as 2,6-dialkylnaphthalenes can be oxidised to naphthalene-2,6-dicarboxylic acid, used to prepare the commercially valuable poly(ethylene naphthalenedicarboxylate) (PEN), which is increasingly finding use in films, liquid crystal polymers, packaging, coatings and adhesives. ${ }^{11}$ A convenient process for preparation of naphthalene-2,6-dicarboxylic acid would involve selective dimethylation of naphthalene followed by oxidation. However, although this method has been investigated, double methylation of naphthalene is not easy to contro and can result in up to ten dimethylnaphthalene isomers, which need separation. ${ }^{12,13}$ Some improvements in selectivity can be made by use of zeolites, but there is still difficulty in obtaining the 2,6-isomer in preference to the 2,7-isomer. For example, it was found that $\beta$-selectivity could be achieved over zeolite HZSM-5, whereas non-selective alkylation was seen over the larger pore H-Mordenite (HM) and HY zeolites. ${ }^{14}$ However, even using HZSM-5 a poor yield of the 2,6-isomer was obtained and the ratio of 2,6/2,7 isomers was approximately 1 .

Isopropylation of naphthalene with propene, ${ }^{15}$ isopropanol, ${ }^{16}$ and isopropyl bromide ${ }^{17}$ as reagents, has been studied using a variety of zeolites. In this case, $\beta, \beta$-selectivities of over $75 \%$, with a $2,6 / 2,7$ ratio of approximately 2 , have been reported using $\mathrm{HM},{ }^{15}$ and the best isopropylation procedure gave a $54 \%$ yield of 2,6-diisopropylnaphthalene with a 2,6/2,7

$\dagger$ Permanent address: Department of Chemistry, Faculty of Science, Tanta University, Tanta 31527, Egypt. ratio of $4 .{ }^{18}$ Cyclohexyl and tert-butyl groups are even bulkier than the isopropyl group. The best reported cyclohexylations ${ }^{19}$ and tert-butylations ${ }^{20}$ of naphthalene make use of HY zeolite, and result in 43 and $28 \%$ yields of the corresponding 2,6-dialkylnaphthalene, with 2,6/2,7 ratios of 1.2 and 5.9, respectively, with the added advantage of easy separation of the 2,6-isomers by crystallisation. It has also been reported that isooctane effects tert-butylation, ${ }^{21}$ and that naphthalene can be tert-butylated using pillared clay catalysts. ${ }^{22}$

At the outset of the work reported here, despite the intensive effort, highly selective production of a 2,6-dialkylnaphthalene remained an elusive goal. Therefore, we undertook a detailed study of the alkylation reaction, especially tert-butylation, to see if improvements could be made in the yield and selectivity for the 2,6-isomer. We have already given a preliminary account of some of the results. ${ }^{9}$ We now report the full details of the successful production of 2,6-di-tert-butylnaphthalene, which can be easily separated from the reaction mixture by crystallisation.

\section{Results and discussion}

Initially, a range of different solids was screened for efficacy in the tert-butylation of naphthalene (1) in cyclohexane, under conditions as close as possible to those used by Moreau and coworkers. ${ }^{20}$ The properties of the commercial zeolites used in this study are recorded in Table 1. The major products of the tertbutylation reaction were 2-tert-butylnaphthalene (2), 2,6-ditert-butylnaphthalene (3) and 2,7-di-tert-butylnaphthalene (4) (Scheme 1) and the yields obtained are given in Table 2.

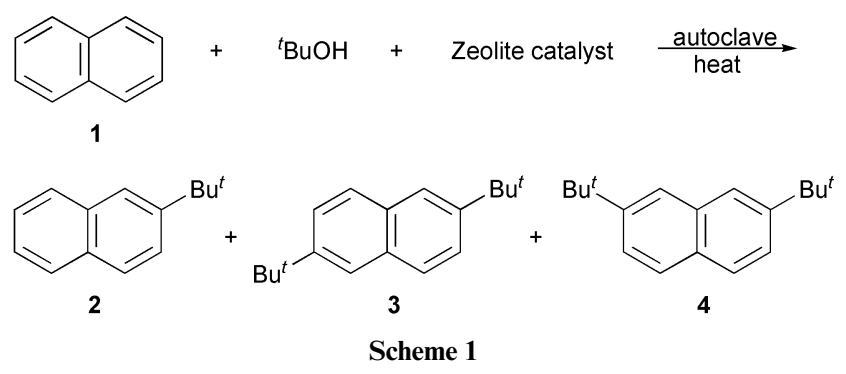

The trends in the results were consistent with the reported ones, ${ }^{20}$ where direct comparison was possible. The minor variations in the absolute results can be understood in terms of the use of different zeolite samples and a different autoclave with different dimensions, resulting in a different self-generated 
Table 1 Typical properties of the commercial zeolites

\begin{tabular}{|c|c|c|c|c|c|}
\hline Zeolite & Product code & $\mathrm{Si} / \mathrm{Al}$ ratio & Nominal cation form & $\mathrm{Na}_{2} \mathrm{O}\left(\mathrm{wt}^{\circ} \%\right)$ & Surface area $/ \mathrm{m}^{2} \mathrm{~g}^{-1}$ \\
\hline HY & CBV 720 & 15 & $\mathrm{H}$ & 0.03 & 780 \\
\hline HY & CBV 760 & 30 & $\mathrm{H}$ & 0.03 & 720 \\
\hline HY & CBV 780 & 40 & $\mathrm{H}$ & 0.03 & 780 \\
\hline $\mathrm{H} \beta$ & CP $814 B-25$ & 12.5 & $\mathrm{NH}_{4}$ & 0.05 & 720 \\
\hline HM & CBV 20A & 10 & $\mathrm{NH}_{4}^{4}$ & 0.02 & 500 \\
\hline HM & CBV $30 A$ & 17.5 & $\mathrm{NH}_{4}$ & 0.02 & 600 \\
\hline HZSM-5 & CBV 5020E & 25 & $\mathrm{H}$ & 0.05 & 425 \\
\hline
\end{tabular}

Table 2 tert-Butylation of naphthalene (1) over various solid catalysts according to Scheme $1^{a}$

\begin{tabular}{|c|c|c|c|c|c|}
\hline \multirow[b]{2}{*}{ Catalyst (Si/Al ratio or HPA wt $\%$ loading) } & \multirow[b]{2}{*}{ Conversion $(\%)^{b}$} & \multicolumn{3}{|c|}{ Yields $(\%)^{b}$} & \multirow[b]{2}{*}{$3 / 4$ ratio $^{c}$} \\
\hline & & 2 & 3 & 4 & \\
\hline HY (15) & 89 & 39 & 33 & 12 & 2.7 \\
\hline $\mathrm{HY}(15)^{d}$ & 84 & 42 & 27 & 11 & 2.5 \\
\hline $\mathrm{HY}(15)^{e}$ & 79 & 39 & 25 & 10 & 2.5 \\
\hline HY (30) & 89 & 41 & 30 & 11 & 2.8 \\
\hline HY (40) & 87 & 41 & 28 & 10 & 2.9 \\
\hline $\mathrm{H} \beta(12.5)$ & 49 & 46 & 2 & 2 & 1.1 \\
\hline $\mathrm{HM}(10)$ & 24 & 22 & 2 & - & - \\
\hline HM (17.5) & 31 & 22 & 6 & 0.4 & 14.0 \\
\hline HZSM-5 (25) & 0 & - & - & - & - \\
\hline MCM-41 (7) & 10 & 9 & 0.2 & 0.1 & 2.0 \\
\hline $\operatorname{MMS}(10)^{g}$ & 49 & 40 & 6 & 3 & 1.9 \\
\hline $\operatorname{HPA}(-)^{h}$ & 0.3 & 0.1 & 0.2 & - & - \\
\hline MCM-41/HPA (30) & 30 & 27 & 2 & 1 & 1.5 \\
\hline MMS/HPA (30) & 48 & 39 & 5 & 4 & 1.4 \\
\hline MMS/HPA (50) & 53 & 43 & 6 & 4 & 1.5 \\
\hline MMS/HPA (70) & 53 & 43 & 6 & 4 & 1.5 \\
\hline
\end{tabular}

${ }^{a} 2 \mathrm{~h}$ stirred autoclave reaction at $160{ }^{\circ} \mathrm{C}$; catalyst $(0.5 \mathrm{~g})$, cyclohexane $(100 \mathrm{ml})$, naphthalene $(1.28 \mathrm{~g} ; 10 \mathrm{mmol})$, tert-butanol $(1.48 \mathrm{~g}$; $20 \mathrm{mmol})$. ${ }^{b}$ Determined by GC. Numbers expressed as percentages have been rounded to the nearest whole number, except numbers that are $<1$ (expressed to 1 decimal place). ${ }^{c}$ The $\mathbf{3 / 4}$ ratio is a direct comparison of GC peak areas. ${ }^{d}$ Reaction took place in chloroform. ${ }^{e}$ Reaction took place in hexane. ${ }^{f}$ MCM-41 (a Mobil composition of matter) was synthesised according to a literature procedure. ${ }^{23}{ }^{g}$ MMS (a mesoporous molecular sieve) was synthesised according to a literature procedure. ${ }^{24}{ }^{h}$ Silicotungstic acid hydrate, $\mathrm{H}_{4} \mathrm{SiW}_{12} \mathrm{O}_{40}$, was used as the heteropolyacid (HPA).

pressure, and different temperature control. Thus, HY was the most active solid and $H \beta$ was essentially non-selective for the production of 3 over 4 (3/4 ratio of 1.1). In addition, we found that HZSM-5 gave no reaction, presumably because the pores were too small to allow the reaction to occur. With a mesoporous molecular sieve (H-MMS), reaction occurred with a naphthalene conversion similar to that with $\mathrm{H} \beta$ but with higher yield and a surprisingly higher $\mathbf{3 / 4}$ ratio of 1.9 . However, attention was attracted mostly by the results with HM. Although with $\mathrm{HM}(\mathrm{Si} / \mathrm{Al} 10)$ only a $24 \%$ conversion of naphthalene and a $2 \%$ yield of di-tert-butylnaphthalene was achieved, it was promising that the only dialkyl isomer detected under these conditions was 3. With HM (Si/Al 17.5), a naphthalene conversion of $31 \%$ and a $6.4 \%$ yield of dialkyl products with a $\mathbf{3} / \mathbf{4}$ ratio of 14 were observed. Therefore, we undertook a more detailed study of the reaction with HM as a catalyst in the hope of enhancing the conversion into dialkylnaphthalenes while retaining the higher selectivity.

Initially we attempted to increase the conversion into desirable product by adding more catalyst. The reaction conditions were kept constant, but the quantity of $\mathrm{HM}(\mathrm{Si} / \mathrm{Al} 10)$ catalyst was increased in stages to $4.0 \mathrm{~g}$. The results obtained are given in Table 3.

It was found that doubling the amount of catalyst to $1.0 \mathrm{~g}$ increased the conversion by $12 \%$ and the yield of di-tert-butylnaphthalenes by $4 \%$. A $3 / 4$ ratio of 17.3 was obtained, already a significant improvement over all previously reported selectivities. When the amount of the HM catalyst was increased to 4.0 $\mathrm{g}$, the conversion of naphthalene and yield of dialkyl products were higher still, but the $\mathbf{3 / 4}$ ratio was not so high (7.0).

In order to gauge the effect of temperature, the reaction temperature was varied in $20^{\circ} \mathrm{C}$ stages from 140 to $220^{\circ} \mathrm{C}$ over $2.0 \mathrm{~g}$ of HM (10). The results obtained are given in Table 4.
It was found that when the temperature was increased to $200{ }^{\circ} \mathrm{C}$, the conversion and the yield of $\mathbf{3}$ were increased from 40 to $52 \%$ and from 5 to $10 \%$, respectively. However, when the temperature was increased further to $220{ }^{\circ} \mathrm{C}$, both conversion and yield of 3 were seen to fall, to $42 \%$ and $8 \%$, respectively. In addition, the yield of $\mathbf{4}$ was seen to increase with every increase in temperature, and as a result the $\mathbf{3 / 4}$ ratio was seen to fall from 22.8 at $140{ }^{\circ} \mathrm{C}$, to 6.4 at $220{ }^{\circ} \mathrm{C}$. Therefore, our results are consistent with the findings of Moreau, who had attributed the decrease in selectivity and conversion to secondary reactions such as dealkylation, disproportionation and transalkylation occurring at elevated temperatures. ${ }^{20}$ Although the conversion and yield increased up to $200{ }^{\circ} \mathrm{C}$, it was decided to standardise the temperature at $180{ }^{\circ} \mathrm{C}$ for further reactions, to preserve good selectivity as well as yield.

To determine if there was any benefit to be gained by varying the amount of the reagent, the amount of tert-butanol was varied over $4.0 \mathrm{~g}$ of $\mathrm{HM}$ at $180^{\circ} \mathrm{C}$. The amount of tert-butanol was increased in stages from 20 to $530 \mathrm{mmol}$. The results obtained are given in Table 5.

It was found that increasing the amount of tert-butanol up to $80 \mathrm{mmol}$ brought about increases in both conversion and the yield of 3, although excessive amounts (more than $80 \mathrm{mmol}$ ) caused both to fall. However, the $\mathbf{3 / 4}$ ratio was seen to increase with increasing tert-butanol without reaching a maximum. For the following studies $4.0 \mathrm{~g}$ of HM and $80 \mathrm{mmol}$ of tert-butanol were chosen.

A series of experiments in which the duration of the reaction was varied from $1 \mathrm{~h}$ to $24 \mathrm{~h}$ in cyclohexane $(100 \mathrm{ml})$ was conducted next. The results obtained are given in Table 6 .

The general trend on increasing the reaction time was an increase in the conversion and yield of $\mathbf{3}$, with little variation in the $\mathbf{3 / 4}$ ratio. However, the conversion increased from $54 \%$ to 
Table 3 Varying the quantity of $\mathrm{HM}(\mathrm{Si} / \mathrm{Al} 10)$ catalyst in the tert-butylation of naphthalene (1) ${ }^{a}$

\begin{tabular}{|c|c|c|c|c|c|}
\hline \multirow[b]{2}{*}{ Quantity of HM (Si/Al 10)/g } & \multirow[b]{2}{*}{ Conversion $(\%)^{b}$} & \multicolumn{3}{|c|}{ Yields $(\%)^{b}$} & \multirow[b]{2}{*}{$3 / 4$ ratio $^{c}$} \\
\hline & & 2 & 3 & 4 & \\
\hline 0.5 & 24 & 22 & 2 & - & - \\
\hline 1 & 36 & 30 & 6 & 0.4 & 17.3 \\
\hline 2 & 37 & 30 & 7 & 0.5 & 12.1 \\
\hline 4 & 51 & 39 & 8 & 1 & 7.0 \\
\hline
\end{tabular}

${ }^{a} 2 \mathrm{~h}$ stirred autoclave reaction at $160{ }^{\circ} \mathrm{C} ; \mathrm{HM}$, cyclohexane $(100 \mathrm{ml})$, naphthalene $(1.28 \mathrm{~g} ; 10 \mathrm{mmol})$, tert-butanol (1.48 g; $\left.20 \mathrm{mmol}\right) .{ }^{b}$ See footnote $b$ to Table 2. ${ }^{c}$ See footnote $c$ to Table 2 .

Table 4 Varying the temperature in the tert-butylation of naphthalene (1) over HM (Si/Al 10, $2.0 \mathrm{~g})^{a}$

\begin{tabular}{llllll}
\hline & & \multicolumn{2}{l}{${\text { Yields }(\%)^{b}}$} \\
\cline { 3 - 5 }$T /{ }^{\circ} \mathrm{C}$ & Conversion $(\%)^{b}$ & $\mathbf{2}$ & $\mathbf{3}$ & $\mathbf{4}$ & \multirow{2}{*}{$\mathbf{3 / 4}$ ratio $^{c}$} \\
\hline 140 & 40 & 33 & 5 & 0.2 & 22.8 \\
160 & 43 & 34 & 8 & 0.5 & 16.4 \\
180 & 47 & 38 & 9 & 0.6 & 13.4 \\
200 & 52 & 41 & 10 & 1 & 9.5 \\
220 & 42 & 31 & 8 & 1 & 6.4
\end{tabular}

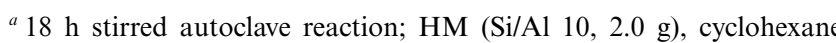
$(100 \mathrm{ml})$, naphthalene $(1.28 \mathrm{~g} ; 10 \mathrm{mmol})$, tert-butanol $(1.48 \mathrm{~g}$; $20 \mathrm{mmol}) .{ }^{b}$ See footnote $b$ to Table $2 .{ }^{c}$ See footnote $c$ to Table 2.

Table 5 Varying the quantity of tert-butanol in the tert-butylation of naphthalene (1) over HM (Si/Al 10, $4.0 \mathrm{~g})^{a}$

\begin{tabular}{|c|c|c|c|c|c|}
\hline \multirow[b]{2}{*}{ tert-Butanol $/ \mathrm{mmol}$} & \multirow[b]{2}{*}{ Conversion $(\%)^{b}$} & \multicolumn{3}{|c|}{ Yields $(\%)^{b}$} & \multirow[b]{2}{*}{$3 / 4$ ratio $^{c}$} \\
\hline & & 2 & 3 & 4 & \\
\hline 20 & 54 & 40 & 12 & 1 & 10.9 \\
\hline 40 & 56 & 40 & 16 & 0.5 & 33.9 \\
\hline 80 & 57 & 38 & 19 & 0.4 & 44.2 \\
\hline 160 & 50 & 37 & 12 & 0.2 & 75.6 \\
\hline 530 & 21 & 19 & 3 & - & - \\
\hline
\end{tabular}

${ }^{a} 18 \mathrm{~h}$ stirred autoclave reaction at $180{ }^{\circ} \mathrm{C} ; \mathrm{HM}(\mathrm{Si} / \mathrm{Al} 10,4.0 \mathrm{~g})$ cyclohexane $(100 \mathrm{ml})$, naphthalene $(1.28 \mathrm{~g} ; 10 \mathrm{mmol})$, tert-butanol. ${ }^{b}$ See footnote $b$ to Table $2 .{ }^{c}$ See footnote $c$ to Table 2 .

Table 6 Varying the reaction time in the tert-butylation of naphthalene (1) over HM (Si/Al 10, $4.0 \mathrm{~g})^{a}$

\begin{tabular}{rlllll}
\hline & & \multicolumn{2}{l}{${\text { Yields }(\%)^{b}}$} & \\
\cline { 3 - 5 }$t / \mathrm{h}$ & Conversion $(\%)^{b}$ & $\mathbf{2}$ & $\mathbf{3}$ & $\mathbf{4}$ & \multirow{3}{*}{$\mathbf{3 / 4}$ ratio $^{c}$} \\
\hline 1 & 54 & 40 & 14 & 0.4 & 38.1 \\
3 & 54 & 41 & 14 & 0.3 & 43.7 \\
6 & 57 & 41 & 15 & 0.4 & 40.7 \\
12 & 57 & 40 & 17 & 0.4 & 41.5 \\
18 & 59 & 40 & 18 & 0.4 & 44.2 \\
24 & 66 & 43 & 22 & 0.5 & 42.7
\end{tabular}

${ }^{a}$ Stirred autoclave reaction at $180^{\circ} \mathrm{C} ; \mathrm{HM}(\mathrm{Si} / \mathrm{Al} 10,4.0 \mathrm{~g})$, cyclohexane $(100 \mathrm{ml})$, naphthalene $(1.28 \mathrm{~g} ; 10 \mathrm{mmol})$, tert-butanol $(5.93 \mathrm{~g}$; $80 \mathrm{mmol}) .{ }^{b}$ See footnote $b$ to Table $2 .{ }^{c}$ See footnote $c$ to Table 2.

$66 \%$ and the yield of 3 from $14 \%$ to $22 \%$ on varying the reaction time from 1 to $24 \mathrm{~h}$. Clearly, the majority of the reaction occurred within the first hour and only an extra $12 \%$ conversion occurred in the next $23 \mathrm{~h}$. Therefore, $1 \mathrm{~h}$ is a more suitable reaction time.

As the conversion and yield of $\mathbf{3}$ were improved by increasing the amount of catalyst and tert-butanol, we planned to give further improvement by adding even more. However, this would involve adding more than $8.0 \mathrm{~g}$ of the valuable HM catalyst. To combat this, an attempt was made at multistaging the reaction,
Table 7 Multistaging tert-butylation of naphthalene (1) over HM $(\mathrm{Si} / \mathrm{Al} 10,4.0 \mathrm{~g})$ in cyclohexane $(100 \mathrm{ml})$ for $18 \mathrm{~h}^{a}$

\begin{tabular}{llllll}
\hline & & \multicolumn{3}{l}{${\text { Yields }(\%)^{b}}$} \\
\cline { 3 - 5 } Stage & Conversion $(\%)^{b}$ & $\mathbf{2}$ & $\mathbf{3}$ & $\mathbf{4}$ & 3/4 ratio $^{c}$ \\
\hline 1 & 60 & 41 & 18 & 0.4 & 44.4 \\
2 & 75 & 40 & 34 & 0.8 & 41.6 \\
3 & 84 & 30 & 50 & 1 & 40.8 \\
$4^{d}$ & 96 & 34 & 47 & 4 & 13.2
\end{tabular}

${ }^{a}$ Each stage is an $18 \mathrm{~h}$ stirred autoclave reaction at $180{ }^{\circ} \mathrm{C}$; catalyst $(4.0 \mathrm{~g})$, cyclohexane $(100 \mathrm{ml})$, naphthalene $(1.28 \mathrm{~g} ; 10 \mathrm{mmol})$, tert-butanol $(5.93 \mathrm{~g} ; 80 \mathrm{mmol})$. At the beginning of each stage, the old

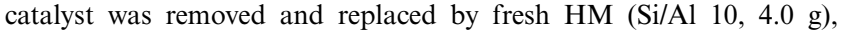
fresh cyclohexane $(100 \mathrm{ml})$ and fresh tert-butanol $(5.93 \mathrm{~g} ; 80 \mathrm{mmol})$. ${ }^{b}$ See footnote $b$ to Table $2 .{ }^{c}$ See footnote $c$ to Table $2 .{ }^{d}$ The yield of by-products has become significant $(>10 \%)$.

such that a smaller portion of the catalyst could be used at a time, allowing recycling of the catalyst to be used, if necessary. After each stage, the old catalyst was removed; the product mixture was concentrated; and fresh catalyst, alkylating agent and solvent were added. The first stage was equivalent to the normal $4 \mathrm{~g} / 80 \mathrm{mmol}$ reaction. The resulting product mixture was reacted again with fresh catalyst, additional tert-butanol (80 mmol) and fresh solvent. Therefore after two stages, HM (10) $(8 \mathrm{~g})$ and tert-butanol $(160 \mathrm{mmol})$ had been reacted with the naphthalene $(10 \mathrm{mmol})$. This process was then repeated for third and fourth stages, such that catalyst (16 g) and tert-butanol $(320 \mathrm{mmol})$ were used in total. The results obtained are given in Table 7.

After the second stage, both the conversion and yield of $\mathbf{3}$ were increased (by 15 and 16\%, respectively), whilst the selectivity was essentially unaltered. After the third stage, the conversion and yield of $\mathbf{3}$ were again increased and the selectivity was still unaltered. After the fourth stage, however, the yield of $\mathbf{3}$ was slightly reduced and the $\mathbf{3 / 4}$ ratio was dramatically lowered. In addition, after the fourth stage the proportions of other products had become significant. Therefore, it would seem that it is possible to push the reaction too far, presumably by causing dealkylation, disproportionation, transalkylation and/ or further reaction.

We attempted to vary the alkylating agent and the volume of solvent in order to increase the conversion into the desired product (3). The results obtained are given in Table 8 .

The volume of cyclohexane was varied from 0 to $200 \mathrm{ml}$. It was found that increasing the solvent from the original volume $(100 \mathrm{ml})$ to $200 \mathrm{ml}$, resulted in a lower conversion (by $13 \%$ ), lower yield of $\mathbf{3}$ (by $8 \%$ ) and a lower $\mathbf{3 / 4}$ ratio (from 44.2 to 36.4). Clearly, dilution of the reaction mixture reduces the rate of reaction. In addition, the pressure generated within the autoclave was increased to $200-250$ psi when $200 \mathrm{ml}$ of cyclohexane was used (typically 150-200 psi for the $100 \mathrm{ml}$ reaction). Reducing the volume of cyclohexane from $100 \mathrm{ml}$ to $50 \mathrm{ml}$ increased the conversion (by $8 \%$ ), yield of $\mathbf{3}(9 \%)$ and $\mathbf{3 / 4}$ ratio (from 44.2 to 45.1 ). Reducing further to $25 \mathrm{ml}$ gave essentially the same conversion and yield of $\mathbf{3}$ as the $50 \mathrm{ml}$ reaction, but 
Table 8 Varying the alkylating agent and the solvent in the tert-butylation of naphthalene (1) over $\mathrm{HM}(\mathrm{Si} / \mathrm{Al} 10,4.0 \mathrm{~g})^{a}$

\begin{tabular}{|c|c|c|c|c|c|c|}
\hline \multirow[b]{2}{*}{ Alkylating agent } & \multirow[b]{2}{*}{ Solvent $/ \mathrm{ml}$} & \multirow[b]{2}{*}{ Conversion $(\%)^{b}$} & \multicolumn{3}{|c|}{ Yields $(\%)^{b}$} & \multirow[b]{2}{*}{$3 / 4$ ratio $^{c}$} \\
\hline & & & 2 & 3 & 4 & \\
\hline tert-Butanol & - & 63 & 30 & 18 & 7 & 2.6 \\
\hline tert-Butanol & Cyclohexane (10) & 79 & 26 & 48 & 1 & 36.3 \\
\hline tert-Butanol & Cyclohexane (25) & 66 & 36 & 26 & 0.4 & 56.6 \\
\hline tert-Butanol & Cyclohexane (50) & 65 & 38 & 28 & 0.6 & 45.1 \\
\hline tert-Butanol & Cyclohexane (100) & 57 & 38 & 19 & 0.4 & 44.2 \\
\hline tert-Butanol & Cyclohexane (200) & 44 & 33 & 11 & 0.3 & 36.4 \\
\hline tert-Butanol & Isooctane $(10)$ & 79 & 24 & 49 & 2 & 23.8 \\
\hline tert-Butanol & Isooctane $(100)$ & 81 & 34 & 43 & 2 & 24.4 \\
\hline tert-Butanol & Isooctane-cyclohexane $(100)^{d}$ & 67 & 35 & 31 & 0.9 & 35.6 \\
\hline tert-Butanol & Octane (100) & 87 & 30 & 48 & 2 & 22.2 \\
\hline Isooctane & Isooctane $(100)$ & 81 & 48 & 17 & 7 & 2.6 \\
\hline Isooctane & Cyclohexane (100) & 0 & - & - & - & - \\
\hline Octane & Octane (100) & 0 & - & - & - & - \\
\hline tert-Butyl methyl ether & Cyclohexane (100) & 47 & 37 & 10 & 0.5 & 20.5 \\
\hline Di-tert-butyl ether & Cyclohexane (100) & 49 & 37 & 10 & 0.8 & 12.9 \\
\hline
\end{tabular}

${ }^{a} 18 \mathrm{~h}$ stirred autoclave reaction at $180^{\circ} \mathrm{C} ; \mathrm{HM}$ (Si/Al 10, $\left.4.0 \mathrm{~g}\right)$, solvent, naphthalene (1.28 g; $\left.10 \mathrm{mmol}\right)$, alkylating agent $(80 \mathrm{mmol}) .{ }^{b}$ See footnote $b$ to Table 2. ${ }^{c}$ See footnote $c$ to Table $2 .{ }^{d}$ Isooctane $(50 \mathrm{ml})$ and cyclohexane $(50 \mathrm{ml})$.

with increased selectivity (45.1 to 56.6). When the volume of cyclohexane was reduced further still to $10 \mathrm{ml}$, the $\mathbf{3 / 4}$ ratio was seen to fall to 36.3 , but the conversion (by 13\%) and yield of 3 (by $22 \%$ ) were significantly increased. When no solvent was present in the reaction mixture, the conversion (by 16\%), yield of $\mathbf{3}(30 \%)$ and $\mathbf{3} / \mathbf{4}$ ratio (from 36.3 to 2.6 ) were all significantly reduced (Table 8 ).

With isooctane as the solvent, the reaction was significantly more rapid than the reaction with cyclohexane as a solvent, but less selective for the production of the 2,6-isomer. In Table 8, the results of reactions with cyclohexane and isooctane as solvents have been compared with a reaction in which the two solvents were mixed. The values of conversion, yield and selectivity for this mixed solvent reaction were essentially intermediate between those for the two reactions in the pure solvents. Therefore, addition of isooctane to cyclohexane is a useful way of improving the yield without reducing the selectivity too significantly. Although further mixed solvent reactions were not attempted, one could predict an increased yield but reduced selectivity if the proportion of isooctane to cyclohexane were increased, and vice versa.

When isooctane was used as alkylating agent in cyclohexane as solvent, no reaction occurred. Also, with octane as both alkylating agent and solvent, no reaction occurred. However, when isooctane was used as both alkylating agent and solvent, as noted above, the reaction occurred at a reasonable rate, but with poor 2,6/2,7-selectivity.

When tert-butyl methyl ether was used as alkylating agent in cyclohexane $47 \%$ conversion, a $10 \%$ yield of 3 and a $\mathbf{3} / \mathbf{4}$ ratio of 20.5 were obtained. Similar conversion and yield of $\mathbf{3}$ were obtained when di-tert-butyl ether was used as alkylating agent, but the $\mathbf{3 / 4}$ ratio was only 12.9 .

The results of the experiments recorded in Table 8 therefore suggest that the reaction takes place most efficiently when a relatively small volume of cyclohexane is used as solvent. Poor results were obtained in the absence of solvent.

Multistaging under the improved conditions over $4.0 \mathrm{~g}$ of HM (10) was therefore attempted. The results obtained are given in Table 9.

Following completion of the second stage, both conversion of naphthalene and yield of $\mathbf{3}$ were increased by $20 \%$ to give $92 \%$ conversion and a $63 \%$ yield of $\mathbf{3}$, the highest yield of $\mathbf{3}$ so far. Moreover, the selectivity was practically unaffected from that after the first stage (34.8 compared with 37.1 from the first stage). After the third stage, total conversion of naphthalene had been virtually achieved, but the yield of $\mathbf{3}$ was not improved. In addition, the $\mathbf{3 / 4}$ ratio had been significantly reduced. After the fourth stage, there was no improvement in
Table 9 Multistaging tert-butylation of naphthalene (1) over HM (Si/Al 10, $4.0 \mathrm{~g})$ in cyclohexane $(10 \mathrm{ml})$ for $1 \mathrm{~h}^{a}$

\begin{tabular}{llllll}
\hline & & \multicolumn{2}{l}{${\text { Yields }(\%)^{b}}$} & \\
\cline { 3 - 5 } Stage & Conversion $(\%)^{b}$ & $\mathbf{2}$ & $\mathbf{3}$ & $\mathbf{4}$ & 3/4 ratio $^{c}$ \\
\hline 1 & & & & & \\
$2^{d}$ & 72 & 21 & 43 & 1 & 37.1 \\
$3^{d}$ & 92 & 13 & 63 & 2 & 34.8 \\
$4^{d}$ & 96 & 13 & 62 & 3 & 25.1 \\
& 97 & 14 & 61 & 3 & 19.1
\end{tabular}

${ }^{a}$ Each stage is a $1 \mathrm{~h}$ stirred autoclave reaction at $180{ }^{\circ} \mathrm{C}$; catalyst $(4.0 \mathrm{~g})$, cyclohexane $(10 \mathrm{ml})$, naphthalene $(1.28 \mathrm{~g} ; 10 \mathrm{mmol})$, tert-butanol $(5.93$ $\mathrm{g} ; 80 \mathrm{mmol})$. At the beginning of each stage, the old catalyst was removed and replaced by fresh $\mathrm{HM}(\mathrm{Si} / \mathrm{Al} 10,4.0 \mathrm{~g})$, fresh cyclohexane $(10 \mathrm{ml})$ and fresh tert-butanol $(5.93 \mathrm{~g} ; 80 \mathrm{mmol}) .{ }^{b}$ See footnote $b$ to Table 2. ${ }^{c}$ See footnote $c$ to Table 2. ${ }^{d}$ See footnote $d$ to Table 7.

Table 10 Multistaging tert-butylation of naphthalene (1) over HM $(\mathrm{Si} / \mathrm{Al} 17.5,4.0 \mathrm{~g})$ in cyclohexane $(10 \mathrm{ml})$ for $1 \mathrm{~h}^{a}$

\begin{tabular}{llllll}
\hline & & \multicolumn{2}{l}{${\text { Yields }(\%)^{b}}$} \\
\cline { 3 - 5 } Stage & Conversion $(\%)^{b}$ & $\mathbf{2}$ & $\mathbf{3}$ & $\mathbf{4}$ & \multirow{3}{*}{$3 / \mathbf{4}$ ratio $^{c}$} \\
\hline 1 & 84 & 18 & 50 & 0.8 & 58.5 \\
$2^{d}$ & 96 & 10 & 60 & 1 & 50.6 \\
$3^{d}$ & 96 & 8 & 60 & 1 & 44.7
\end{tabular}

${ }^{a}$ See footnote $a$ to Table $8 .{ }^{b}$ See footnote $b$ to Table 2. ${ }^{c}$ See footnote $c$ to Table $2 .{ }^{d}$ See footnote $d$ to Table 7 .

the yield of $\mathbf{3}$, and the $\mathbf{3 / 4}$ ratio was again reduced as significantly more 4 was obtained. Therefore, it would seem that after two stages, the reaction had essentially stopped and the only effect of further stages was to diminish the $\mathbf{3 / 4}$ ratio.

In order to check on the possibility of reuse of the zeolite, a single reaction was carried out as above, and the zeolite was recovered following extraction of the products and was then regenerated by heating overnight in air in an oven set at $550{ }^{\circ} \mathrm{C}$. The yield and selectivity of an alkylation reaction conducted in an identical manner to that described above, but using the recovered zeolite, gave essentially identical results. Indeed, repeated recovery and reuse of the zeolite gave essentially the same results for six reuses, after which the activity and selectivity began to fall.

Another study was conducted using the $\mathrm{HM}(\mathrm{Si} / \mathrm{Al}=17.5)$ zeolite (Table 10). As a result, we found that the optimum conditions for maximising the yield of $\mathbf{3}$ while retaining selectivity over this zeolite involved two successive $1 \mathrm{~h}$ autoclave reactions 
Table 11 tert-Butylation of naphthalene (1) over various catalysts under the optimised conditions ${ }^{a}$

\begin{tabular}{|c|c|c|c|c|c|}
\hline \multirow[b]{2}{*}{ Catalyst (Si/Al) } & \multirow[b]{2}{*}{ Conversion $(\%)^{b}$} & \multicolumn{3}{|c|}{ Yields $(\%)^{b}$} & \multirow[b]{2}{*}{$3 / 4$ ratio $^{c}$} \\
\hline & & 2 & 3 & 4 & \\
\hline HM (10) & 72 & 21 & 43 & 1 & 37.1 \\
\hline HM $(17.5)^{d}$ & 84 & 18 & 50 & 0.8 & 58.5 \\
\hline $\mathrm{HY}(15)^{d}$ & 94 & 12 & 37 & 18 & 2.0 \\
\hline $\mathrm{H} \beta(12.5)^{d}$ & 87 & 29 & 19 & 19 & 1.0 \\
\hline
\end{tabular}

${ }^{a} 1 \mathrm{~h}$ stirred autoclave reaction at $180{ }^{\circ} \mathrm{C}$; catalyst $(4.0 \mathrm{~g})$, cyclohexane $(10 \mathrm{ml})$, naphthalene $(1.28 \mathrm{~g} ; 10 \mathrm{mmol})$, tert-butanol $(5.93 \mathrm{~g} ; 80 \mathrm{mmol})$.

${ }^{b}$ See footnote $b$ to Table $2 .{ }^{c}$ See footnote $c$ to Table $2 .{ }^{d}$ See footnote $d$ to Table 7 .

at $180{ }^{\circ} \mathrm{C}$, using $\mathrm{HM}(4.0 \mathrm{~g}, \mathrm{Si} / \mathrm{Al}$ ratio 17.5$)$, tert-butanol $(80 \mathrm{mmol})$ and cyclohexane $(10 \mathrm{ml})$ for each stage and an initial $10 \mathrm{mmol}$ of naphthalene (Table 10).

Under these conditions, a $50 \%$ yield of $\mathbf{3}$ and a $\mathbf{3 / 4}$ ratio of 58.5 were achieved after the first stage, a $60 \%$ yield with a 50.6 ratio after the second stage, and a $60 \%$ yield with a 44.7 ratio after the third stage. The optimum product mixture, in addition to $61 \%$ in total of di-tert-butylnaphthalenes (3) and (4), contained $4 \%$ of residual naphthalene (1), $10 \%$ of 2-tert-butylnaphthalene (2) and significant quantities of several other 2,6dialkylnaphthalenes. The GC analysis of the product mixture after the second stage showed that 2-tert-amyl-6-tert-butylnaphthalene and 2,6-di-tert-amylnaphthalene were obtained in 7 and $4 \%$ yields, respectively. In addition, small quantities $(3 \%)$ of tri-tert-butylnaphthalenes were detected. Approximately $85 \%$ of 3 present in the product could be obtained by concentrating the reaction mixture following the optimum reaction and then allowing the product to crystallise. Purification was obtained by crystallisation from hot ethanol, mp $148^{\circ} \mathrm{C}$ (lit., ${ }^{25}$ $147-148{ }^{\circ} \mathrm{C}$ ). Separation of the remaining 3 ( $\mathrm{ca}$. 15\% of the total) was obtained by Kugelrohr distillation under reduced pressure.

For comparison, reactions with $\mathrm{HY}$ and $\mathrm{H} \beta$ were conducted under the conditions optimised for HM, though only for a single stage reaction. The results obtained are given in Table 11 .

The results confirmed that $\mathrm{HY}$ and $\mathrm{H} \beta$ remain more active than HM under these conditions. In the case of HY, the total yield of $\mathbf{3}$ and $\mathbf{4}$ obtained (55\%) was also higher than with HM, but the results confirmed the significance of the HM catalyst for obtaining selectivity as HY achieved a 3/4 ratio of only 2 and $\mathrm{H} \beta$ proved to be non-selective. Therefore, the actual yield of 3 was significantly higher with HM $(17.5)(50 \%)$ than with HM (10) $(43 \%)$, HY (37\%) or H $\beta(19 \%)$. Also, the absence of significant quantities of other di-tert-butylnaphthalene isomers would make its separation from the mixture much easier.

Water is a by-product formed in the alkylation reaction if an alcohol such as tert-butanol is used as the alkylating agent. ${ }^{26}$ Interestingly, water has shown beneficial effects on both activity and selectivity in certain circumstances. It was of interest to determine if a further increase in activity and/or selectivity could be achieved by the addition of water. Therefore in Table
Table 13 Effect of the dealumination of the HM catalyst in the tertbutylation of naphthalene $(\mathbf{1})^{a}$

\begin{tabular}{llllll}
\hline & & \multicolumn{2}{l}{${\text { Yields }(\%)^{b}}$} & \\
\cline { 3 - 5 } Treatment & Conversion $(\%)^{b}$ & $\mathbf{2}$ & $\mathbf{3}$ & $\mathbf{4}$ & \multirow{2}{*}{$\mathbf{3} / \mathbf{4}$ ratio $^{c}$} \\
\hline- & 72 & 21 & 43 & 1 & 37 \\
\hline $4 \mathrm{M} \mathrm{HCl}^{d}$ & 69 & 19 & 41 & 0.8 & 49 \\
$8 \mathrm{M} \mathrm{HCl}^{d}$ & 54 & 15 & 31 & 0.2 & 140
\end{tabular}

${ }^{a} 1 \mathrm{~h}$ stirred autoclave reaction at $180{ }^{\circ} \mathrm{C}$; HM (4.0 g), cyclohexane $(10 \mathrm{ml})$, naphthalene $(1.28 \mathrm{~g} ; 10 \mathrm{mmol})$, tert-butanol $(5.93 \mathrm{~g} ; 80 \mathrm{mmol})$. ${ }^{b}$ See footnote $b$ to Table 2. ${ }^{c}$ See footnote $c$ to Table $2 .{ }^{d}$ Dealuminated by stirring with acid for $24 \mathrm{~h}$ at $85^{\circ} \mathrm{C}$.

12 , the results are given for reactions with various amounts of added water. The theoretical volume of water that can be generated from tert-butanol $(80 \mathrm{mmol})$ is $c a .1 .4 \mathrm{ml}$. For all reactions reported in Table 12, the combined volume of cyclohexane and added water was kept constant at $10 \mathrm{ml}$, and reactions were attempted in which the solvent composition varied between $100 \%$ cyclohexane and $100 \%$ water.

Little reaction ( $4 \%$ conversion and $2 \%$ yield of 3 ) was achieved with $10 \mathrm{ml}$ of water as solvent, whereas the reaction in $10 \mathrm{ml}$ cyclohexane achieved high activity $(72 \%$ conversion and a $43 \%$ yield of 3). Due to the poor conversion in water, 4 was not detected in the product mixture, hence a $\mathbf{3 / 4}$ ratio could not be determined. With a $1 / 1$ cyclohexane and water mix, slight improvements in conversion and yield of $3(11 \%$ and $6 \%$, respectively) were obtained, although still a $\mathbf{3 / 4}$ ratio could not be determined. With a $3 / 1$ cyclohexane and water mix, significant reaction occurred ( $61 \%$ conversion and a $37 \%$ yield of $\mathbf{3}$ ), although still not to the extent of the $10 \mathrm{ml}$ cyclohexane reaction. In addition the selectivity was not high $(3 / 4$ ratio $=21.7)$. When a 9/1 mix was used, comparable conversion and yield of 3 to those in the $10 \mathrm{ml}$ cyclohexane reaction were obtained, although the selectivity was still significantly lower (23.3 compared with 37). Hence the addition of water proved ineffective at improving either the activity or the selectivity of the reaction.

We have previously seen that $\mathrm{HM}$ with a higher $\mathrm{Si} / \mathrm{Al}$ ratio gave higher conversion, yield of $\mathbf{3}$ and selectivity. Therefore, attempts were made to dealuminate a sample of HM (10) in the hope of improvement. Two batches of dealuminated HM were prepared by leaching of $\mathrm{HM} \mathrm{(10)} \mathrm{with} \mathrm{HCl}$; the first batch was treated with $4 \mathrm{M} \mathrm{HCl}$ and the second with $8 \mathrm{M} \mathrm{HCl}$. Reactions with the two dealuminated HM catalysts were conducted, and compared against non-dealuminated HM (Table 13).

In essence, the $\mathrm{HM}$ dealuminated with $4 \mathrm{M} \mathrm{HCl}$ gave an identical conversion and yield of $\mathbf{3}$ to the non-dealuminated sample. However, the $\mathbf{3 / 4}$ ratio was significantly increased from 37 to 49 , indicating that some dealumination must have occurred. As the selectivity obtained was between that obtained over commercial HM (10) and HM (17.5), it is likely that the $\mathrm{Si} / \mathrm{Al}$ ratio of the dealuminated sample was between 10 and 17.5, though this was not checked. The results achieved over $\mathrm{HM}$ dealuminated with $8 \mathrm{M} \mathrm{HCl}$ indicate that substantial

Table 12 Effect of the addition of water in the tert-butylation of naphthalene (1) over $\mathrm{HM}(\mathrm{Si} / \mathrm{Al} 10,4.0 \mathrm{~g})^{a}$

\begin{tabular}{|c|c|c|c|c|c|}
\hline \multirow[b]{2}{*}{ Cyclohexane/water (ml) } & \multirow[b]{2}{*}{ Conversion $(\%)^{b}$} & \multicolumn{3}{|c|}{ Yields $(\%)^{b}$} & \multirow[b]{2}{*}{$3 / 4$ ratio $^{c}$} \\
\hline & & 2 & 3 & 4 & \\
\hline $10 / 0$ & 72 & 21 & 43 & 1 & 37.1 \\
\hline $9 / 1$ & 73 & 15 & 45 & 2 & 23.3 \\
\hline $7.5 / 2.5$ & 61 & 18 & 37 & 2 & 21.7 \\
\hline $5 / 5$ & 11 & 3 & 6 & - & - \\
\hline $0 / 10$ & 4 & 2 & 2 & - & - \\
\hline
\end{tabular}

${ }^{a} 1 \mathrm{~h}$ stirred autoclave reaction at $180{ }^{\circ} \mathrm{C} ; \mathrm{HM}(\mathrm{Si} / \mathrm{Al} 10,4.0 \mathrm{~g})$, solvent $(10 \mathrm{ml})$, naphthalene $(1.28 \mathrm{~g} ; 10 \mathrm{mmol})$, tert-butanol $(5.93 \mathrm{~g} ; 80 \mathrm{mmol})$. ${ }^{b}$ See footnote $b$ to Table 2. ${ }^{c}$ See footnote $c$ to Table 2 . 
Table 14 Cyclohexylation of naphthalene (1) over various catalysts according to Scheme $2\left(\mathrm{R}=\mathrm{C}_{6} \mathrm{H}_{11}\right)^{a}$

\begin{tabular}{|c|c|c|c|c|c|c|}
\hline \multirow[b]{2}{*}{ Catalyst (Si/Al) } & \multirow[b]{2}{*}{ Alkylating agent } & \multirow[b]{2}{*}{ Conversion $(\%)^{b}$} & \multicolumn{3}{|c|}{ Yields $(\%)^{b}$} & \multirow[b]{2}{*}{ 6/7 ratio $^{c}$} \\
\hline & & & 5 & 6 & 7 & \\
\hline HY (15) & Cyclohexene & 95 & 47 & 24 & 16 & 1.5 \\
\hline HY (15) & Cyclohexanol & 96 & 52 & 16 & 10 & 1.6 \\
\hline HY (15) & Cyclohexyl bromide & 94 & 50 & 20 & 14 & 1.4 \\
\hline $\mathrm{H} \beta(12.5)$ & Cyclohexene & 18 & 16 & 1 & 1 & 1.0 \\
\hline HM (10) & Cyclohexene & 0 & - & - & - & - \\
\hline H-ZSM-5 (25) & Cyclohexene & 0 & - & - & - & - \\
\hline MMS/HPA (30) & Cyclohexene & 65 & 45 & 2 & 1 & 2.0 \\
\hline MMS/HPA (50) & Cyclohexene & 71 & 49 & 2 & 1 & 2.0 \\
\hline MMS/HPA (70) & Cyclohexene & 71 & 50 & 2 & 2 & 1.3 \\
\hline
\end{tabular}

${ }^{a} 0.5 \mathrm{~h}$ stirred autoclave reaction at $200{ }^{\circ} \mathrm{C}$; catalyst $(1.0 \mathrm{~g})$, cyclohexane $(50 \mathrm{ml})$, naphthalene $(0.64 \mathrm{~g} ; 5 \mathrm{mmol})$, alkylating agent $(10 \mathrm{mmol})$. ${ }^{b}$ See footnote $b$ to Table $2 .{ }^{c}$ See footnote $c$ to Table 2 .

dealumination had occurred. The selectivity was drastically increased from 37 to 140 on dealumination. However, the conversion and yield of $\mathbf{3}$ were significantly lower than those obtained over the non-dealuminated sample. This suggests either that few acid sites remained in the solid due to a significant proportion of the aluminium having being removed and/or that the amount of extra-framework alumina deposited in the pores was sufficient to cause significant hindrance to diffusion. The results suggest that there is a balance to be struck between maximising the conversion and yield of $\mathbf{3}$ and maximising the selectivity. For this study, we decided to continue with the non-dealuminated samples of HM (10).

Varying the volume of the solvent altered the pressure generated within the autoclave, as well as changing the concentrations of the materials contained therein. Therefore, a study was conducted to determine if pressure was an important factor in the reaction. A $10 \mathrm{ml}$ reaction with self-generated pressure (50-100 psi) was compared against a reaction where the pressure was maintained with nitrogen at a much higher level (500 psi). It was found that there was a slight increase of $4 \%$ in both the conversion $(76 \%)$ and yield of $3(47 \%)$ when nitrogen pressure was applied. However, the selectivity fell from 37 to 12.4 . Clearly, the increased pressure was disadvantageous as significantly more $\mathbf{4}$ was formed. This probably results from an increased likelihood of all collisions under the higher pressure, although the effect of the small reduction in molecular volume of the transition state may have contributed to the readier formation of $\mathbf{4}$ in the pores under such higher pressures.

In all reactions thus far, the reactants and catalysts were mixed directly at the start. It was decided to investigate dropwise addition of the tert-butanol to see how this would affect the yield of $\mathbf{3}$ and/or the selectivity. For this purpose the autoclave was fitted with a suitable dropping funnel and the tertbutanol was introduced over a period of $1 \mathrm{~h}$. The results showed that there was little difference in conversion and yield of 3 between the drip-fed reaction and the reaction when all reactants were added at the start. However, the selectivity was considerably lower (3/4 ratio of 9.7 compared with 37 ) when tert-butanol was drip-fed, which is perhaps not surprising considering the previous findings with a large amount of catalyst and only a small proportion of alkylating agent present throughout. When a standard reaction was carried out and then further tert-butanol was added and the heating continued, a higher conversion $(87 \%)$ and a significantly higher yield of $3(63 \%)$ was achieved than in the simple reaction. The $\mathbf{3 / 4}$ ratio was 16.0. As the dropwise addition of tert-butanol was disadvantageous, an attempt was made to drip-feed the naphthalene (in $10 \mathrm{ml}$ cyclohexane) into the reaction mixture. The selectivity achieved was similar to that obtained in the standard reaction, but the rate of reaction was dramatically reduced, giving only $12 \%$ conversion and a $3 \%$ yield of 3 after $1.5 \mathrm{~h}$. As neither the dropwise addition of naphthalene nor tert-butanol alone was beneficial, simultaneous dropwise addition of both reagents to the catalyst was attempted. The results showed that the activity was significantly lower (a conversion of $43 \%$ compared with $72 \%$ and a $25 \%$ yield of 3 compared with $43 \%$ ) than if the reagents and catalysts were present from the start of the reaction, although the selectivity was similar.

All tert-butylation reactions of naphthalene carried out thus far were under autoclave conditions, so it was of interest to see if the tert-butylation could be achieved in open reflux. It was found that tert-butylation could be achieved under reflux conditions but not to the same extent as under autoclave conditions. Performing the reaction using a Dean-Stark apparatus was beneficial to the reaction, but the reflux reaction was still inferior to the autoclave reaction. An attempt to use a higher boiling tert-butylating agent (tert-butyl octyl ether) provided no advantage.

In our tert-butylation study, we have shown that 2,6-dialkylnaphthalene can be produced in high yield and selectivity. One of the reasons for studying the tert-butylation reaction in detail was the easy separation of the desired product. The cyclohexylation of naphthalene also benefits from this easy separation of the 2,6-isomer from the product mixture. A preliminary study was therefore conducted into naphthalene cyclohexylation in the hope of improving the selectivity of the 2,6-isomer. Cyclohexylation reactions at $200{ }^{\circ} \mathrm{C}$, using solid catalyst $(1.0 \mathrm{~g})$, alkylating agent $(10 \mathrm{mmol})$ and solvent $(50 \mathrm{ml})$ for an initial $5.0 \mathrm{mmol}$ of naphthalene (1) were attempted (Scheme 2, where $\mathrm{R}=\mathrm{C}_{6} \mathrm{H}_{11}$ ). The results obtained are given in Table 14.<smiles>[R]c1cccc2ccccc12</smiles>

5 Alkylating agent + Zeolite catalyst $\underset{\text { autoclave }}{\text { heat }}$

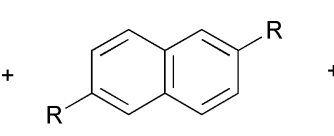

6<smiles>[R]c1ccc2ccc([R])cc2c1</smiles>

7
$\mathrm{R}=\mathrm{C}_{6} \mathrm{H}_{11},{ }^{i} \mathrm{Pr},{ }^{n} \mathrm{Bu},{ }^{i} \mathrm{Bu},{ }^{\mathrm{sec}} \mathrm{Bu}$

Scheme 2

It is clear that zeolite HY is significantly more active in the cyclohexylation than any other zeolites tried. Reactions with cyclohexene, cyclohexanol and cyclohexyl bromide as alkylating agents were also attempted. It was found that all three cyclohexylation reagents achieved similar conversions ( $\mathrm{ca}$. 95\% naphthalene conversion). However, cyclohexanol was less $\beta, \beta-$ selective than cyclohexene and cyclohexyl bromide. $\mathrm{H} \beta$ gave only $18 \%$ conversion and $2 \%$ of dicyclohexylnaphthalenes (6) and (7), and akin to the $H \beta$ catalysed tert-butylation reaction, failed to achieve 2,6-selectivity (6/7 ratio of 1.0$)$. Unsurprisingly, HM did not give a reaction under those conditions, pre- 
Table 15 Alkylation of naphthalene (1) using various alkylating agents over HM (Si/Al 10, 4.0 g) according to Scheme $2^{a}$

\begin{tabular}{|c|c|c|c|c|c|c|}
\hline \multirow[b]{2}{*}{ Alkylating agent } & \multirow[b]{2}{*}{$\mathrm{R}$} & \multirow[b]{2}{*}{ Conversion $(\%)^{b}$} & \multicolumn{3}{|c|}{ Yields $(\%)^{b}$} & \multirow[b]{2}{*}{$6 / 7$ ratio $^{c}$} \\
\hline & & & 5 & 6 & 7 & \\
\hline Isopropanol & Isopropyl & 13 & 12 & 0.5 & 0.2 & 2.2 \\
\hline 1-Butanol & $n$-Butyl & 3 & 2 & 0.1 & 0.1 & 1.0 \\
\hline 2-Butanol & sec-Butyl & 31 & 24 & 4 & 1 & 3.3 \\
\hline Isobutanol & Isobutyl & 5 & 3 & 0.3 & 0.2 & 1.5 \\
\hline
\end{tabular}

${ }^{a} 1 \mathrm{~h}$ stirred autoclave reaction at $180{ }^{\circ} \mathrm{C} ; \mathrm{HM}(\mathrm{Si} / \mathrm{Al} 10,4.0 \mathrm{~g})$, cyclohexane $(10 \mathrm{ml})$, naphthalene $(1.28 \mathrm{~g} ; 10 \mathrm{mmol})$, alkylating agent $(10 \mathrm{mmol})$. ${ }^{b}$ See footnote $b$ to Table $2 .{ }^{c}$ See footnote $c$ to Table 2 .

sumably more forcing conditions being required for a reaction to occur. In addition, no reaction was achieved over H-ZSM-5 due to the pores being too small to allow the reaction to occur.

Cyclohexylation was also attempted over synthesised mesoporous zeolites. In Table 13, the cyclohexylation results over MMS with a $30 \mathrm{wt} \%$ loading of a heteropolyacid (HPA) are compared against solids with higher loading (50 wt $\%$ and $70 \mathrm{wt} \%$ ), to determine if the conversion and selectivity are affected by increased HPA in the pores of the solid. It was found that the conversion was increased by $6 \%$ when the loading was increased to $50 \mathrm{wt} \%$ loading; however, there was no change in the yield of $6, \beta, \beta$-selectivity or $6 / 7$ ratio. When the loading was increased further to $70 \mathrm{wt} \%$, the $6 / 7$ ratio was reduced from 2.0 to 1.3 . Therefore, we conclude that increasing the loading within the pores of the mesoporous support has little beneficial effect upon the activity or selectivity.

Several other alkylations of naphthalene at $180{ }^{\circ} \mathrm{C}$, using $\mathrm{HM}(\mathrm{Si} / \mathrm{Al}$ ratio of $10,4.0 \mathrm{~g})$, alkylating agent $(10 \mathrm{mmol})$ and cyclohexane $(10 \mathrm{ml})$ for an initial $10 \mathrm{mmol}$ of naphthalene have been attempted (Scheme 2). The results obtained are given in Table 15.

With isopropanol as alkylating agent, a naphthalene conversion of $13 \%$ was achieved. The yield of 6 was only $0.5 \%$, although a 6/7 ratio of 2.2 was achieved, which is consistent with results obtained by others using $\mathrm{HM}$ with a moderate $\mathrm{Si} /$ Al ratio. ${ }^{15}$ For the primary alcohols (1-butanol and isobutanol) it was not surprising that naphthalene conversions and selectivities were poor ( 3 and 5\% yields, respectively, with $6 / 7$ ratios of 1.0 and 1.5 , respectively). It is clear that the carbocation formed has a major effect on the activity and selectivity. It is not surprising that tert-butanol is therefore the most active and most selective, although it should be recognized that the reaction conditions are optimised for this reagent and not for the others. However, it is promising that moderate selectivity can be achieved with the secondary alcohol, 2-butanol (31\% conversion with a $6 / 7$ ratio of 3.3 ).

These reaction systems are extremely complex, involving inter alia: several phases (solid, liquid and gas); reactions that may take place inside or outside of the zeolite pores; competing reactions, some of which may involve equilibria; and the generation of by-products (e.g. water, alkene) that may themselves influence the reaction course. It would be of interest to study them in greater detail in order better to understand the mechanism and the role played by such parameters as added water or higher pressure.

\section{Experimental}

A Hewlett Packard HP 5890 (Series II) gas chromatography, fitted with a capillary column RTX-1 (100\% dimethylpolysiloxane; $30 \mathrm{~m}, 0.32 \mathrm{~mm}$ ID) was used to analyse reaction mixtures. The $\mathrm{GC}$ conditions used for analysis were: $100{ }^{\circ} \mathrm{C}$ for

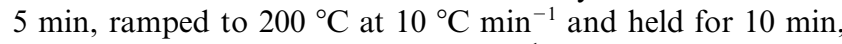
then ramped to $250{ }^{\circ} \mathrm{C}$ at $5{ }^{\circ} \mathrm{C} \mathrm{min}-1$ and held for $30 \mathrm{~min}$. Tetradecane was used as an internal standard.

\section{Chemicals}

Cyclohexane (99+\%) was obtained from Fischer Scientific and HPLC grade tert-butanol $(99.5+\%)$, scintillation grade naphthalene $(99+\%)$ and other chemicals were obtained from Aldrich. All were used directly without further purification.

\section{Zeolites}

Commercial zeolites were purchased from Aldrich Chemical Company or provided as gifts by Zeolyst International. MCM-41 and H-MMS were synthesised by literature procedures. ${ }^{23,24}$ All catalysts were freshly calcined prior to the reaction by heating in air at $550{ }^{\circ} \mathrm{C}$ for a minimum of $6 \mathrm{~h}$. Those having ammonium cations would be converted into the corresponding proton forms during the calcination process.

\section{Typical experimental procedure}

Quantities are recorded in the footnotes to the appropriate tables. All reactions were carried out in a $450 \mathrm{ml}$ glass-lined Parr autoclave, fitted with a thermocouple, gauge block assembly and (sometimes) liquid charging pipette. A magnetic stirrer was used.

To the glass liner of the autoclave were added a magnetic bar, naphthalene, alkylating agent and solvent. The mixture was allowed to stir until all naphthalene had dissolved ( $c a .5 \mathrm{~min}$ ) and then the catalyst was added. The glass liner was transferred into the autoclave, which was sealed and heated for the appropriate reaction time and temperature, with stirring, under self-generated pressure. In a few experiments the system was artificially pressurised to 500 psi with nitrogen gas prior to heating.

After the given reaction time, the heating device was removed and the autoclave was allowed to cool to room temperature $(c a .2 \mathrm{~h})$. The apparatus was then washed thoroughly with acetone and the catalyst was removed by filtration. The solid was thoroughly extracted with acetone and the mother liquor was concentrated.

For the reactions that involved multistages, after the above procedure the product mixture was concentrated and fresh catalyst, tert-butanol and solvent were added. The reaction was then allowed to proceed again under the same conditions.

\section{Conclusion}

The autoclave reaction of tert-butanol with naphthalene at $180{ }^{\circ} \mathrm{C}$ in the presence of sufficient HM zeolite provides a convenient, high yielding and highly regioselective method for the synthesis of 2,6-di-tert-butylnaphthalene. Furthermore, heating easily regenerates the zeolite, which can be reused up to six times to give results similar to those of a fresh sample. Although this method is easily the most selective yet discovered for production of a 2,6-dialkylnaphthalene, it may be difficult to oxidise the tert-butyl groups to carboxy groups. ${ }^{27}$ Also, there would be a loss of six carbon atoms during this process, which is wasteful. Therefore, there is still room for considerable improvement in the clean and selective synthesis of naphthalene-2,6-dicarboxylic acid. 


\section{Acknowledgements}

We thank the EPSRC and the University of Wales Swansea for financial support, and the EPSRC Mass Spectrometry Centre at Swansea for GC-MS analyses. We also thank the EPSRC, the Higher Education Funding Council for Wales (ELWa-HEFCW) and the University of Wales Swansea for grants that enabled the purchase and upgrading of NMR equipment used in the course of this work, and Zeolyst International for gifts of zeolites. G. A. El-Hiti thanks the Royal Society of Chemistry for an international author grant.

\section{References}

1 Chemistry of Waste Minimization, ed. J. H. Clark, Chapman and Hall, London, 1995.

2 See for example: Solid Supports and Catalysts in Organic Synthesis, ed. K. Smith, Ellis Horwood, Chichester, 1992, pp. 130-170; J. M. Thomas and W. J. Thomas, Principles and Practice of Heterogeneous Catalysis, VCH, Weinheim, 1997; J. H. Clark, Catalysis of Organic Reactions using Supported Inorganic Reagents, VCH, New York, 1994; Introduction to Zeolite Science and Practice, ed. H. Van Bekkum, E. M. Flanigan and J. C. Jansen, Stud. Surf. Sci. Catal., 1991, 58; J. H. Clark, S. R. Cullen, S. J. Barlow and T. W. Bastock, J. Chem. Soc., Perkin Trans. 2, 1994, 1117.

3 Electrophilic Aromatic Substitution, ed. R. Taylor, John Wiley and Sons, Chichester, 1990.

4 K. Smith, K. Fry, M. Butters and B. Nay, Tetrahedron Lett., 1989, 30, 5333; K. Smith, A. Musson and G. A. DeBoss, J. Org. Chem., 1998, 63, 8448; K. Smith, T. Gibbins, R. W. Millar and R. P. Claridge, J. Chem. Soc., Perkin Trans. 1, 2000, 2753; K. Smith, S. Almeer and S. J. Black, Chem. Commun., 2000, 1571; K. Smith, S. Almeer and C. Peters, Chem. Commun., 2001, 2748 K. Smith, S. Almeer, S. J. Black and C. Peters, J. Mater. Chem., 2002 12, 3285 .

5 K. Smith, P. He and A. Taylor, Green Chem., 1999, 1, 35; K. Smith and D. Bahzad, Chem. Commun., 1996, 476; K. Smith, G. A.
El-Hiti, M. E. W. Hammond, D. Bahzad, Z. Li and C. Siquet, J. Chem. Soc., Perkin Trans. 1, 2000, 2745.

6 K. Smith, M. Butters and B. Nay, Synthesis, 1985, 1157; K. Smith, M. Butters, W. E. Paget, D. Goubet, E. Fromentin and B. Nay, Green Chem., 1999, 1, 83.

7 K. Smith, Z. Zhenhua and P. K. G. Hodgson, J. Mol. Catal. A, 1998, 134, 121.

8 K. Smith and G. Pollaud, J. Chem. Soc., Perkin Trans. 1, 1994, 3519.

9 K. Smith and S. D. Roberts, Catal. Today, 2000, 60, 227.

10 K. Smith, G. M. Ewart and K. R. Randles, J. Chem. Soc., Perkin Trans. 1, 1997, 1085

11 K. Tanabe and W. F. Hölderich, Appl. Catal. A, 1999, 181, 399.

12 S. F. Newman, J. D. Fellmann and P. H. Kilner, US Pat., US 5003102, 1991 .

13 G. A. Olah and J. A. Olah, J. Am. Chem. Soc., 1976, 98, 1839

14 D. Fraenkel, M. Cherniavsky, B. Ittah and M. Levy, J. Catal., 1986, 101, 273.

15 A. Katayama, M. Toba, G. Takeuchi, F. Mizukami, S.-I. Niwa and S. Mitamura, J. Chem. Soc., Chem. Commun., 1991, 39.

16 C. Song and S. Kirby, Micropor. Mater., 1994, 2, 467.

17 P. Moreau, A. Finiels, P. Geneste and J. Solofo, J. Catal., 1992, 136, 487.

18 P. P. B. Notte, G. M. J. L. Poncelet, M. J. H. Remy, P. E. M. G. Lardinois and M. J. M. Van Hoecke, Eur. Pat., EP 0528096, 1993.

19 P. Moreau, A. Finiels, P. Geneste, F. Moreau and J. Solofo, J. Org. Chem., 1992, 57, 5040.

20 Z. Liu, P. Moreau and F. Fajula, Appl. Catal. A, 1997, 159, 305

21 E. Armengol, A. Corma, H. García and J. Primo, Appl. Catal. A, 1997, 149, 411.

22 S. Kitabayashi, T. Shindo, K. Ono and H. Ohnuma, Nippon Kagaku Kaishi, 1996, 624 (Chem. Abstr., 1996, 125, 89575m).

23 J. S. Beck, J. C. Vartuli, W. J. Roth, M. E. Leonowicz, C. T. Kresge, K. D. Schmitt, C. T.-W. Chu, D. H. Olson, E. W. Sheppard, S. B. McCullen, J. B. Higgins and J. L. Schlenker, J. Am. Chem. Soc., $1992, \mathbf{1 1 4}, 10834$

24 R. Mokaya and W. Jones, Chem. Commun., 1996, 981.

25 J.-C. Richer, N. Baskevitch, L. Erichomovitch and F. L. Chubb, Can. J. Chem., 1968, 46, 3363.

26 A. D. Schmitz and C. Song, Catal. Lett., 1996, 40, 59

27 S. G. Brandenberger, L. W. Maas and I. Dvortzky, J. Am. Chem. Soc., 1961, 83, 2146. 